\section{Detection of myositis-specific antibodies}

It was with much interest that we read the recent European League Against Rheumatism/American College of Rheumatology classification criteria for idiopathic inflammatory myopathies. ${ }^{1}$ These criteria include Jo-1 autoantibodies, and the authors discussed that future updates of the criteria should also include the more recently identified myositis-specific autoantibodies. ${ }^{12}$ The interest in autoantibodies for classification is also illustrated by a recent proposal for a new clinicoserological classification of adult autoimmune myositis, which is based on the association of autoantibodies with distinct clinical phenotypes. ${ }^{34}$ For example, antibodies to synthetases (eg, Jo-1, PL-7 and PL-12) define the antisynthetase syndrome, anti-MDA-5 antibodies are associated with myositis with overlap features such as interstitial lung disease, anti-TIF- $1 \gamma$ and anti-NXP-2 define a subgroup of dermatomyositis and anti-SRP and anti-HMGCR are associated with necrotising autoimmune myositis. $^{2}$

As autoantibodies play a role in the newly proposed classifications, ${ }^{12}$ it is expected that measurement of myositis-specific autoantibodies will be increasingly introduced in clinical practice. Most of the myositis-specific autoantibodies have been identified by immunoprecipitation. Alternative, easyto-use commercial line/dot immunoassays are available. However, such assays are not standardised and may suffer from low specificity. ${ }^{2}$ Therefore, these assays need to be further validated.

We evaluated a cohort of 144 patients with inflammatory myopathy (IIM) and 240 controls (blood donors, chronic inflammatory demyelinating polyneuropathy, rheumatoid arthritis, systemic sclerosis, Sjögren's syndrome and systemic lupus erythematosus; 40 of each) for myositis-specific autoantibodies using assays from Alphadia (myositis 12 IgG dot for Bluediver) (Mons, Belgium), Euroimmun (Euroline Autoimmune Inflammatory Myopathies) (Lübeck, Germany) and Trinity Biotech (ImmcoStripe Myositis Advanced LIA) (Buffalo, New York, USA).

The results are shown in table 1 . We observed differences in specificity (reactivity in controls) between the manufacturers and between individual antibodies. For example, $2.9 \%$ and $2.4 \%$ of controls tested positive for anti-Jo- 1 by Euroimmun and Trinity, respectively, compared with $0.4 \%$ by Alphadia. Overall, Euroimmun and Trinity showed more reactivity in controls than Alphadia, except for anti-SAE for which Euroimmun showed less reactivity in controls. Differences in reactivities between manufacturers were also observed in myositis patients, with the most pronounced difference for anti-TIF- $1 \gamma$ (2.1\% with Alphadia versus $12.4 \%$ with Euroimmun and $11 \%$ with Trinity). It should be noted that even for an established marker such as antiJo-1 antibodies, differences between manufacturers were observed in patients with IIM. The likelihood ratio (LR) (prevalence of antibodies in patients divided by prevalence of antibodies in controls) gives a good estimate of how the test result affects the post-test probability (an LR $>10$ indicates a clinical significant difference in pretest to posttest probability). The LRs are shown in table 1 and further illustrate differences between individual antibodies and between manufacturers.

Table 2 shows the corresponding phenotype of myositis-specific antibodies in patients with IIM. The association between antisynthetase antibodies and interstitial lung disease, arthritis and Raynaud's phenomenon was highly significant for all assays. The association of TIF- $1 \gamma$ antibodies and dermatomyositis was high for two of the three assays tested. The association of other antibodies with certain phenotypes (eg, association of TIF- $1 \gamma$ and NXP-2 with malignancy, of Mi-2 with dermatomyositis, NXP-2 with calcinosis and MDA-5 with amyopathic IIM) were weaker and differed between the assays (table 2), indicating that the assays did not perform similarly.

Taken together, as myositis-specific autoantibodies are included in classification criteria, it is important that clinicians and laboratory professionals are aware of the performance characteristics of the assays used to detect such antibodies. Initiatives to harmonise assays across manufacturers are needed.

Jean-Baptiste Vulsteke, ${ }^{1}$ Ellen De Langhe, ${ }^{2,3}$ Kristl G Claeys, ${ }^{4,5,6}$ Doreen Dillaerts, ${ }^{7}$ Koen Poesen, ${ }^{8,9}$ Jan Lenaerts, ${ }^{2}$ René Westhovens, ${ }^{2,3}$ Philip Van Damme, ${ }^{4,10}$ Daniel Blockmans, ${ }^{1}$ Petra De Haes, ${ }^{11}$ Xavier Bossuyt ${ }^{7,9}$

'Department of General Internal Medicine, University Hospitals Leuven, Leuven, Belgium

${ }^{2}$ Department of Rheumatology, University Hospitals Leuven, Leuven, Belgium ${ }^{3}$ Laboratory of Tissue Homeostasis and Disease, Skeletal Biology and Engineering Research Center, Department of Development and Regeneration, KU Leuven, Leuven, Belgium

${ }^{4}$ Department of Neurology, University Hospitals Leuven, Leuven, Belgium ${ }^{5}$ Laboratory for Muscle Diseases and Neuropathies, Department of Neurosciences, KU Leuven, Leuven, Belgium

${ }^{6}$ Department of Neurology and Institute of Neuropathology, University Hospital RWTH Aachen, Aachen, Germany

${ }^{7}$ Department of Microbiology and Immunology, Experimental Laboratory Immunology, KU Leuven, Leuven, Belgium

${ }^{8}$ Laboratory for Molecular Neurobiomarker Research, KU Leuven, Leuven, Belgium ${ }^{9}$ Laboratory Medicine, University Hospitals Leuven, Leuven, Belgium

${ }^{10}$ Department of Neurosciences, Experimental Neurology, VIB Center for Brain \& Disease Research, Laboratory of Neurobiology, KU Leuven, Leuven, Belgium

${ }^{11}$ Department of Dermatology, University Hospitals Leuven, Leuven, Belgium

Correspondence to Dr Xavier Bossuyt, Laboratory Medicine, University Hospitals Gasthuisberg, Leuven 3000, Belgium; xavier.bossuyt@uzleuven.be

Acknowledgements We would like to thank Alphadia, Euroimmun and Trinity for providing the reagents to perform this study. PVD holds a senior clinical investigatorship of FWO-Vlaanderen.

Contributors EDL, J-BV, KGC, KP and XB designed the study. J-BV, DD, EDL and $X B$ analysed the data. DD performed the autoantibody assays. EDL, KGC, PDH, JL, $P V D, R W$ and $D B$ take care of the patients included in the study and revised the manuscript. J-BV, EDL and XB drafted the manuscript.

Funding This study was funded by Alphadia, D-tek, Trinity - Immco and Euroimmun.

Competing interests None declared.

Patient consent Retrospective study using leftover samples.

Ethics approval Local Ethics Committee.

Provenance and peer review Not commissioned; internally peer reviewed.

(c) Article author(s) (or their employer(s) unless otherwise stated in the text of the article) 2019. All rights reserved. No commercial use is permitted unless otherwise expressly granted.

$J$-BV and EDL contributed equally.

\section{Check for updates}

To cite Vulsteke J-B, De Langhe E, Claeys KG, et al. Ann Rheum Dis 2019;78:e7.

Received 28 December 2017

Accepted 30 December 2017

Published Online First 25 January 2018

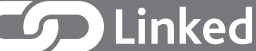

- http://dx.doi.org/10.1136/annrheumdis-2018-212948 


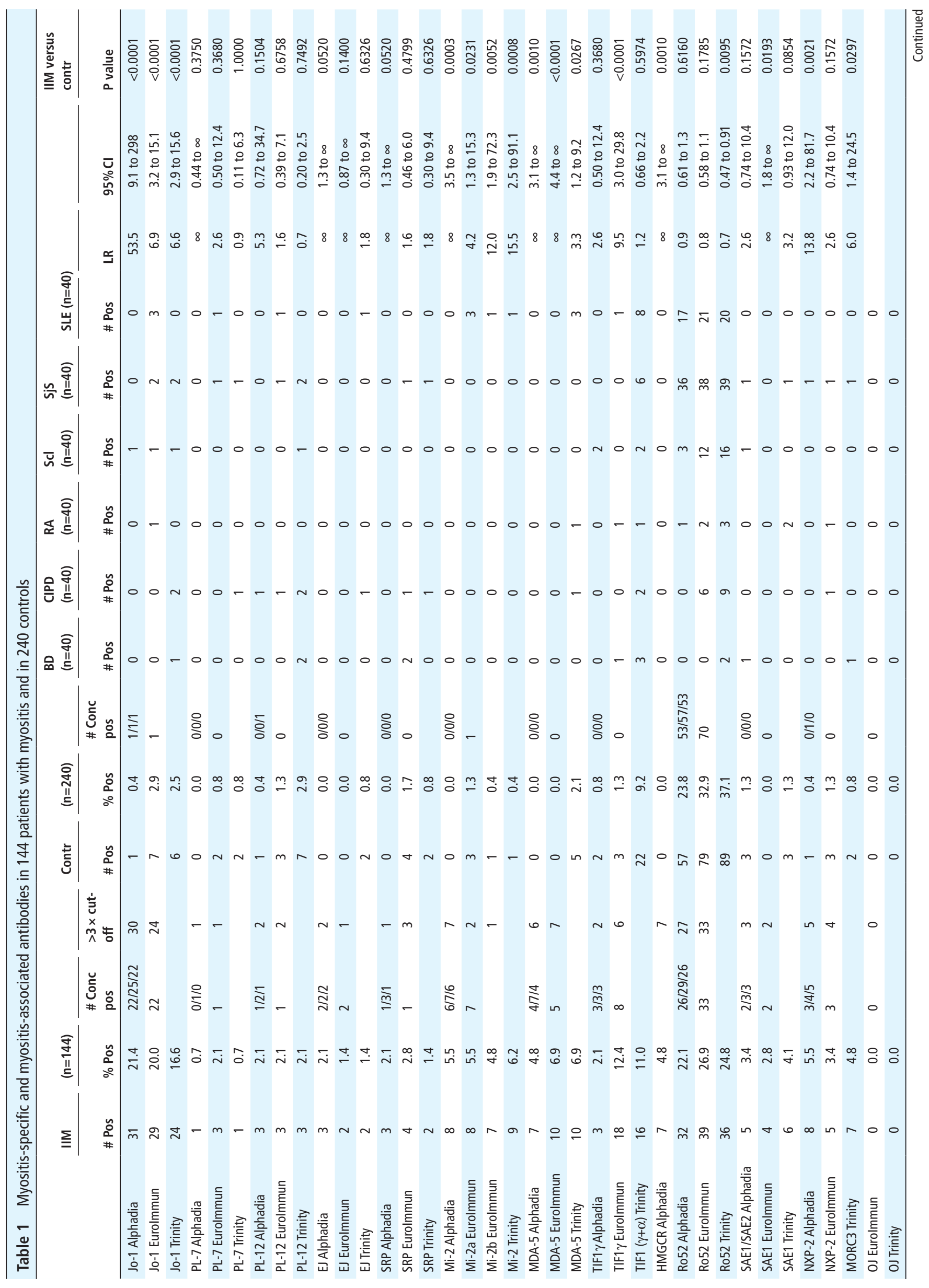




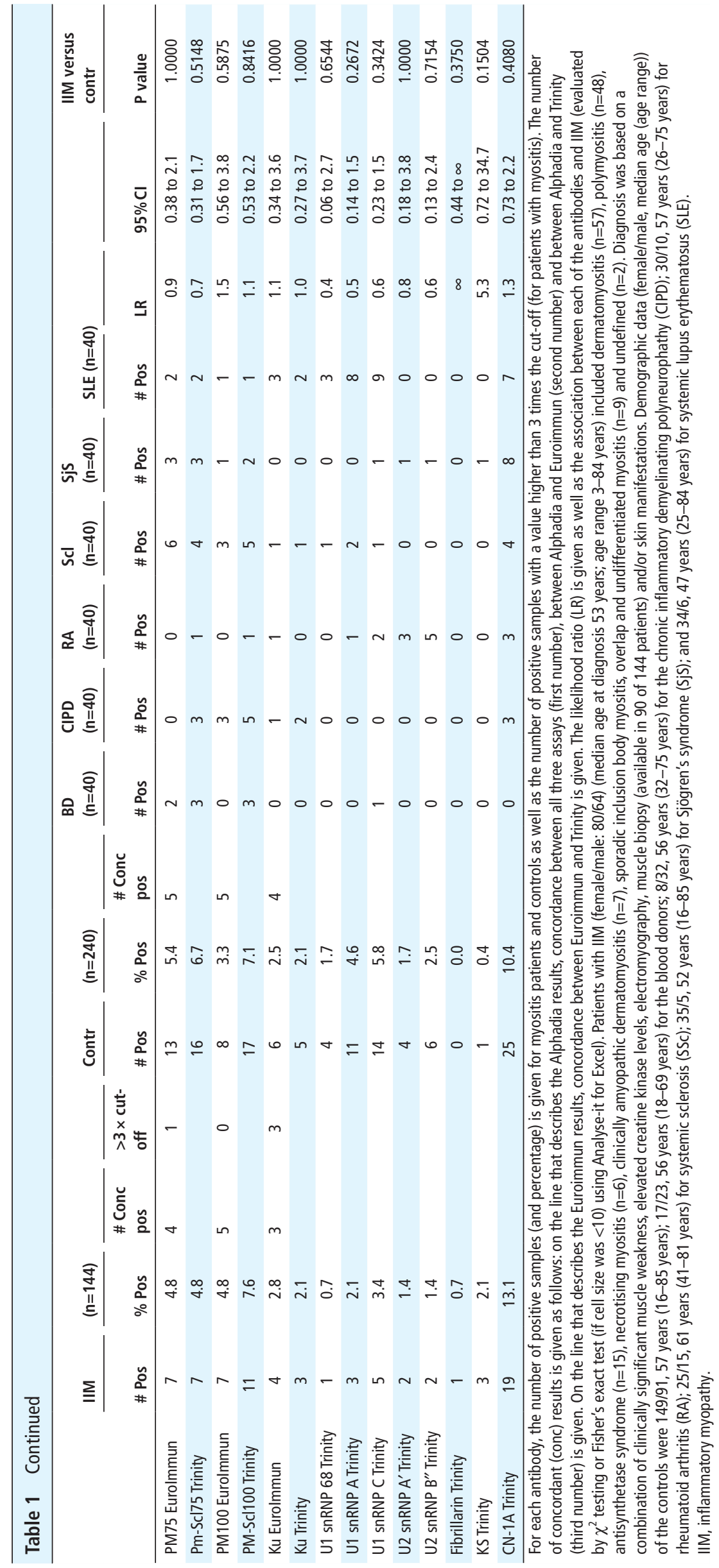


Table 2 Association of myositis-specific antibodies with clinical phenotype in patients with IIM

\begin{tabular}{|c|c|c|c|c|c|c|c|c|c|c|c|c|c|c|c|c|}
\hline \multirow[b]{3}{*}{ Phenotype } & \multirow[b]{3}{*}{ Marker } & \multicolumn{5}{|c|}{ Alphadia } & \multicolumn{5}{|c|}{ Euroimmun } & \multicolumn{5}{|c|}{ Trinity } \\
\hline & & Pos & Pos & Neg & Neg & & Pos & Pos & Neg & Neg & & Pos & Pos & Neg & Neg & \\
\hline & & Pos & Neg & Pos & Neg & $P$ value & Pos & Neg & Pos & Neg & $P$ value & Pos & Neg & Pos & Neg & $P$ value \\
\hline \multirow[t]{2}{*}{ Malignancy } & Tif1- $\gamma$ & 1 & 2 & 10 & 131 & 0.2135 & 3 & 14 & 8 & 119 & 0.1240 & 4 & 12 & 7 & 121 & 0.0212 \\
\hline & NXP-2 (MORC3) & 2 & 6 & 9 & 127 & 0.1155 & 1 & 4 & 10 & 129 & 0.3318 & 1 & 6 & 10 & 127 & 0.4337 \\
\hline \multirow[t]{5}{*}{ DM skin lesions } & $\mathrm{Mi}-2$ & 6 & 2 & 53 & 83 & 0.0638 & 6 & 5 & 53 & 80 & 0.3582 & 5 & 4 & 54 & 81 & 0.4873 \\
\hline & TIF- $\gamma$ & 3 & 0 & 56 & 85 & 0.0667 & 16 & 1 & 43 & 84 & $<0.0001$ & 14 & 2 & 45 & 83 & $<0.0001$ \\
\hline & NXP-2 (MORC3) & 4 & 4 & 55 & 81 & 0.7166 & 3 & 2 & 56 & 83 & 0.4003 & 4 & 3 & 55 & 82 & 0.4449 \\
\hline & MDA-5 & 5 & 2 & 54 & 83 & 0.1228 & 8 & 2 & 51 & 83 & 0.0159 & 6 & 4 & 53 & 81 & 0.3176 \\
\hline & SAE & 4 & 1 & 55 & 84 & 0.1590 & 4 & 0 & 55 & 85 & 0.0265 & 4 & 2 & 55 & 83 & 0.2273 \\
\hline Amyopathic DM & MDA-5 & 2 & 5 & 5 & 132 & 0.0380 & 2 & 8 & 5 & 129 & 0.0758 & 2 & 8 & 5 & 129 & 0.0758 \\
\hline Calcinosis & NXP-2 (MORC3) & 2 & 6 & 7 & 129 & 0.0801 & 2 & 3 & 7 & 132 & 0.0316 & 7 & 5 & 2 & 130 & $<0.0001$ \\
\hline Inclusion body myositis & $\mathrm{CN}-1 \mathrm{~A}$ & & & & & & & & & & & 1 & 18 & 5 & 120 & 0.5791 \\
\hline \multirow[t]{2}{*}{ Arthritis } & Jo-1 & 14 & 17 & 10 & 103 & $<0.0001$ & 13 & 16 & 11 & 104 & $<0.0001$ & 10 & 14 & 14 & 106 & 0.0003 \\
\hline & Jo-1, PL7, PL12 or EJ & 16 & 21 & 8 & 99 & $<0.0001$ & 16 & 21 & 8 & 99 & 0.0001 & 12 & 18 & 12 & 102 & 0.0004 \\
\hline \multirow[t]{2}{*}{ ILD } & Jo-1 & 19 & 12 & 13 & 98 & $<0.0001$ & 19 & 10 & 13 & 100 & $<0.0001$ & 15 & 9 & 17 & 101 & $<0.0001$ \\
\hline & Jo-1, PL7, PL12 or EJ & 22 & 15 & 10 & 95 & $<0.0001$ & 23 & 17 & 9 & 93 & $<0.0001$ & 17 & 13 & 15 & 9797 & $<0.0001$ \\
\hline \multirow[t]{2}{*}{ Raynaud } & Jo-1 & 12 & 18 & 13 & 99 & 0.0003 & 13 & 15 & 12 & 102 & $<0.0001$ & 9 & 14 & 16 & 103 & 0.0063 \\
\hline & Jo-1, PL7, PL12 or EJ & 16 & 20 & 9 & 97 & $<0.0001$ & 17 & 22 & 8 & 95 & $<0.0001$ & 11 & 18 & 14 & 99 & 0.0013 \\
\hline
\end{tabular}

The association was evaluated by $\chi^{2}$ testing or Fisher's exact test (if cell size was $<10$ ) using Analyse-it for Excel.

IIM, inflammatory myopathy.

Ann Rheum Dis 2019;78:e7. doi:10.1136/annrheumdis-2017-212915

\section{REFERENCES}

1 Lundberg IE, Tjärnlund A, Bottai M, et al. European League Against Rheumatism/ American College of Rheumatology Classification Criteria for Adult and Juvenile Idiopathic Inflammatory Myopathies and Their Major Subgroups. Ann Rheum Dis 2017;2017:1955-64.
2 Lundberg IE, Tjärnlund A. Response to: '2017 EULAR/ACR classification criteria for adult and juvenile idiopathic inflammatory myopathies and their major subgroups: little emphasis on autoantibodies, why?' by Malaviya. Ann Rheum Dis 2017. doi: 10.1136/ annrheumdis-2017-212709 (Epub ahead of print: 15 Dec 2017).

3 Malaviya AN. EULAR/ACR classification criteria for adult and juvenile idiopathic inflammatory myopathies and their major subgroups: little emphasis on autoantibodies why? Ann Rheum Dis 2017;212701.

4 Senécal JL, Raynauld JP, Troyanov Y. Editorial: a new classification of adult autoimmune myositis. Arthritis Rheumatol 2017;69:878-84. 